\title{
Upregulation of natural killer cells functions underlies the efficacy of intratumorally injected dendritic cells engineered to produce interleukin-12
}

\author{
Mercedes Rodríguez-Calvillo, Marina Duarte, Iñigo Tirapu, \\ Pedro Berraondo, Guillermo Mazzolini, Chen Qian, Jesús Prieto, and Ignacio Melero \\ Gene Therapy Unit, Department of Internal Medicine, University of Navarra, Pamplona, Spain
}

(Received 29 August 2001; revised 9 November 2001; accepted 30 November 2001)

\begin{abstract}
Objective. Injection of dendritic cells (DC) engineered with recombinant adenoviral vectors to produce interleukin-12 (IL-12) inside experimental murine tumors frequently achieves complete regressions. In such a system the function of $\mathrm{CD8}^{+} \mathrm{T}$ cells has been shown to be an absolute requirement, in contrast to observations made upon depletion of $\mathrm{CD4}^{+} \mathrm{T}$ cells, which minimally affected the outcome. The aim of this work was to study the possible involvement of natural killer (NK) cells in this setting.

Materials, Methods, and Results. Depletions with anti-AsialoGM1 antiserum showed only a small decrease in the proportion of complete regressions obtained that correlated with induction of NK activities in lymphatic tissues into which DC migrate, whereas combined depletions of $\mathrm{CD4}^{+}$and NK cells completely eliminated the antitumor effects. Likewise in vivo neutralization of interferon- $\gamma($ IFN- $\gamma)$ also eliminated those therapeutic effects. Trying to define the cellular role played by NK cells in vivo, it was observed that injection of cultured DC inside the spleen of T- and B-cell-deficient (Rag1 ${ }^{-/}$) mice induced upregulation of NK activity only if DC had been adenovirally engineered to produce IL-12. In addition, identically transfected fibroblasts also activated NK cells, indicating that IL-12 transfection was the unique requirement. Equivalent human DC only activated in vitro the cytolytic and cytokine-secreting functions of autologous NK cells if transfected to express human IL-12.

Conclusions. Overall, these results point out an important role played by NK cell activation in the potent immunotherapeutic effects elicited by intratumoral injection of IL-12-secreting DC and that NK activation under these conditions is mainly, if not only, dependent on IL-12. () 2002 International Society for Experimental Hematology. Published by Elsevier Science Inc.
\end{abstract}

Dendritic cells (DC) are populations of professional antigen-presenting cells that are being used to elicit antitumor immune responses both in rodents and in humans [1-3]. Functionally, DC can progress from an immature state in which they take up and process antigens efficiently but activate $\mathrm{T}$ cells poorly, to a mature state in which such features are inverted $[1,4]$. Maturation is induced by proinflammatory cytokines or certain microbe components [1]. A therapeutically potent treatment fashion is to introduce those cells by direct injection into experimental tumors. Such an approach is only successful if DC are transfected to express interleukin (IL)-12 [5,6], IL-7 [7], IL-2 [8], or CD40L [9],

Offprint requests to: Ignacio Melero, M.D., Ph.D., Facultad de Medicina, Universidad de Navarra, C/Irunlarrea 1, 31008 Pamplona, Spain with the condition that DC share MHC antigen-presenting molecules with the tumor-bearing animals [5]. DC capture antigens from the malignant tissue, possibly by multiple but still ill-defined mechanisms [10-15], and promptly migrate to lymph nodes where they present tumor antigens to $\mathrm{T}$ cells [16]. In the case of DC-secreting IL-12, high production of IFN- $\gamma$ at this location as well as the induction of potent tumor-specific CTL activity has been documented [6].

Interleukin-12 was identified as a factor that activates the function of natural killer (NK) cells $[17,18]$. NK cells express receptors for IL-12 and when stimulated with this cytokine potently upregulate their functions [19], as it has been also observed in vivo in human cancer patients treated systemically with this protein [20]. It is possible that IL-12 acts not only by increasing the level of expression of effector molecules such as perforin and granzymes [17,18,21,22], 
but also by controlling the function or the expression of receptors triggering cytotoxicity [23]. Besides the elegant mechanisms that regulate whether or not engagement with a target cell causes its destruction in vitro [24-26], little is known about the real functions of NK cells in vivo. NKdeficient mice are largely normal [27], and observations in the limited number of patients with congenital NK deficiency only suggest that these cells are involved in the immune response against herpes viruses [28,29]. Their involvement in tumor immunity is controversial, although their contribution in experimental immunotherapy in certain mouse models has been revealed by specific depletion with antibodies [17,30-32].

DC have been reported to regulate NK functions [33], and IL-12 production by DC seems to be the key event at regulating NK, cytotoxic T-lymphocytes (CTL), and Th1 responses $[17,19]$. The upregulation of NK functions that has already been seen in vitro with mature DC requires direct DC-NK contact [33] with the involvement of IL-12 and IL-18 [34]. We show that immature DC, such as those used for intratumoral injection [5,6], do not upregulate NK functions unless transfected with IL-12 and that cultured DC engineered to produce IL-12 potently activated NK cells both in vivo and in vitro. We report a reduction of antitumor activity when NK cells are depleted, probably explained by loss of NK functions upregulated by DC-secreting IL-12. Moreover, complete loss of the therapeutic effect by simultaneous depletion of $\mathrm{NK}$ and $\mathrm{CD}^{+}{ }^{+} \mathrm{T}$ cells point out an activity shared by both lymphocyte subsets that is absolutely required to induce antitumor effects and that is activated by IL-12.

\section{Materials and methods}

\section{Mice and cell lines}

$\mathrm{BALB} / \mathrm{c}, \mathrm{C} 57 \mathrm{BL} / 6$, and $\mathrm{Rag} 1^{-1-}$ in $\mathrm{C} 57 \mathrm{~B} 1 / 6$ background mice were obtained from Harlan (Barcelona, Spain), and used between 6 and 18 weeks of age. $\operatorname{Rag} 1^{-1-}$ mice were bred in our animal facility.

MC38 and CT26 are colon adenocarcinomas of C57BL/6 and $\mathrm{BALB} / \mathrm{c}$ origin, respectively [5]. YAC-1 is a NK cell-sensitive T-cell lymphoma of A/Sn background [31]. The HLA-negative erythroleukemia K562 was purchased from American Type Culture Collection (ATCC, Rockville, MD, USA) and the MHC class I-deficient 721.221 B-LCL was obtained from Dr. López-Botet (Hospital de la Princesa, Madrid, Spain). IZA-1 is an SV40T-transfected immortalized fibroblast line of BALB/c origin [5]. Cells were maintained in RPMI 1640 medium with $2 \mathrm{mM}$ L-glutamine, $100 \mathrm{U} / \mathrm{mL}$ streptomycin, $100 \mu \mathrm{g} / \mathrm{mL}$ penicillin, and $100 \mu \mathrm{g} / \mathrm{mL}$ cyprofloxacin, and supplemented with $10 \%$ heat-inactivated fetal calf serum (FCS). All cell culture reagents were from Gibco (Basel, Switzerland).

\section{Tumor model and in vivo experiments}

For treatment of tumor-bearing mice in vivo, BALB/c mice were inoculated subcutaneously in the right flank with $4 \times 10^{5}$ CT26 cells. On day 7 , tumors were treated and thereafter growth was measured weekly [5].
For intrasplenic injections, Rag1 $1^{-1-}$ mice under general anesthesia underwent laparotomy and $2 \times 10^{6} \mathrm{DC}$ (in $50 \mu \mathrm{L}$ ) were injected inside the spleen with a $28 \mathrm{G}$ syringe.

\section{$\mathrm{CD}^{+} \mathrm{T}$-cell and $\mathrm{NK}$-cell depletion}

L3T4 $\left(\mathrm{CD}^{+}{ }^{+}\right.$-specific rat anti-mouse antibody was obtained as ascitic fluid in nude mice of the hybridoma GK1-5 (ATCC). Depletion of NK cells was accomplished by administration of a rabbit anti-AsialoGM1 antiserum (Wako, Germany). Both antibodies were administered intraperitoneally at a dose of $100 \mu \mathrm{L}$ per dose on day -2 , on day -1 , before dendritic cell administration, and every 7 days thereafter as previously described [35]. Cell-depletion activity by these antibodies has been tested for every batch by FACS in the spleen of control mice. In vivo neutralization of IFN$\gamma$ was achieved by intraperitoneal injection of ascitic fluid of the hybridoma R4-6A2 (ATCC).

Cytokines and reagents

Recombinant human IL-4 was obtained from PeproTech (London, UK), and recombinant human granulocyte-macrophage colony-stimulating factor (GM-CSF) (Leucomax) was from Novartis (Basel, Switzerland). Recombinant mouse IL-4, recombinant murine GM-CSF, recombinant human IL-12, and tumor necrosis factor- $\alpha$ (TNF- $\alpha$ ) were from Peprotech and anti-human IL-12 and anti-mouse IL-12 were obtained from PharMingen (San Diego, CA, USA). Polyclonal rat IgG was purchased from Sigma (Madrid, Spain).

Culture of murine dendritic cells

The method for generation of DC from bone marrow has been previously described [36]. Cells were cultured for seven days in 12well plates (Reactiva, Barcelona, Spain) at $10^{6} / \mathrm{mL}$ in RPMI-1640 medium (Gibco) supplemented with $100 \mathrm{U} / \mathrm{mL}$ penicillin (Life Technologies), $100 \mu \mathrm{g} / \mathrm{mL}$ streptomycin, $2 \mathrm{mM}$ glutamine, $10 \%$ fetal bovine serum (Gibco), $10 \mu \mathrm{g} / \mathrm{mL}$ cyprofloxacin (Vita, Barcelona, Spain), $20 \mathrm{ng} / \mathrm{mL}$ rmIL-4 (PeproTech), and $20 \mathrm{ng} / \mathrm{mL}$ rmGM-CSF (PeproTech). After 3 and 5 days of culture nonadherent cells were removed and fresh medium with cytokines was added. On day 7, cells in suspension and loosely adherent cells were collected.

\section{Culture of human dendritic cells}

Peripheral blood mononuclear cells (PBMC) were isolated from buffy-coats of healthy donors obtained from the Blood Bank of Navarra in Pamplona (Spain) by Ficoll density-gradient centrifugation (Ficoll-Hypaque, Pharmacia, Uppsala, Sweden). Cells from the light-density fraction were enriched with anti-CD14 magnetic beads (Miltenyi Biotech, Bergisch Gladbach, Germany) according to manufacturer's protocols. $\mathrm{CD} 14^{+}$cells were cultured in 6-well plates (Costar) at a density of $10^{6}$ cells $/ \mathrm{mL}$ in the presence of rhGM-CSF (100 ng/mL) and rhIL-4 (100 ng/mL) in RPMI1640 medium (Gibco) supplemented with 10\% FCS (Gibco) and with antibiotics (100 U/mL penicillin, $100 \mu \mathrm{g} / \mathrm{mL}$ streptomycin, $10 \mu \mathrm{g} /$ $\mathrm{mL}$ cyprofloxacin). Fresh media and cytokines were replenished every 2 days according to previously reported protocols [37].

\section{Generation of human $\mathrm{CD}^{2} 6^{+}$cells}

PBMC were isolated by centrifugation on Ficoll-Hypaque (Pharmacia) density gradient from autologous normal donor buffycoats. Mononuclear cells were incubated with magnetic beads coated with anti-CD56 antibodies (MACS, Miltenyi Biotech) and were enriched using positive selection columns as described above. $\mathrm{CD} 6^{+}$cells were employed immediately or were main- 
tained frozen in $10 \%$ dimethylsulfoxide (DMSO)-containing FCS. Coculture of $\mathrm{CD}^{+} 6^{+}$cells with DC was carried out in U-bottom 96-well plates (Costar) at the indicated NK:DC ratios.

\section{Recombinant adenovirus production and adenovirus infection}

The construction of a recombinant adenovirus carrying either murine or human IL-12 (AdCMVmIL-12/AdCMVhIL-12) has been previously reported [38 and C. Qian et al., manuscript in preparation). Adenovirus carrying lacZ (AdCMVLacZ) reporter gene has also been reported [39]. Purified viruses were extensively dialyzed against $10 \mathrm{mM}$ Tris, $1 \mathrm{mM} \mathrm{MgCl}{ }_{2}$ and stored in aliquots at $-80^{\circ} \mathrm{C}$. Plaque-forming units (pfu) were determined by repeated cytopathic plaque assays on 293 cells and contamination with competent adenovirus was excluded by lack of lytic infection of a panel of $\mathrm{E} 1^{-}$cells.

DC were infected on day 7 of culture. Briefly, cells were recovered, washed, and incubated with the adenoviruses at the indicated multiplicities of infection (MOIs) in serum-free medium $\left(5 \times 10^{6}\right.$ cells $/ 500 \mu \mathrm{L}$ ). After 1 hour at $37^{\circ} \mathrm{C}$, complete medium was added (1 mL/10 6 cells).

\section{Immunofluorescence and flow cytometry}

Expression of surface markers was determined by flow cytometry (FACScalibur, Becton-Dickinson, San Jose, CA, USA). After culture, either DCs or NK cells were washed and labeled with FITC, $\mathrm{PE}$, or PerCP-conjugated mouse anti-human mAbs (Becton-Dickinson), antiCD11c, antiCD14, antiCD3, and antiCD56 for $30 \mathrm{~min}$ utes at $4^{\circ} \mathrm{C}$. Nonreactive fluorochrome-tagged mouse mAbs were used as controls (Becton-Dickinson).

DC cultures were FITC-labeled with PKH2 (Sigma) according to manufacturer's instructions and fluorescence microphotographs were taken with a conventional microscope equipped with a UV lamp (Nikon).

Cytotoxicity assays

Cytotoxicity was assessed by standard $4 \mathrm{~h}{ }^{51} \mathrm{Cr}$-release assays [40].

Cytokine quantitation by ELISA

Human IFN- $\gamma$ and IL-12 (OptiEA, PharMingen) and mouse IFN- $\gamma$ (Cytoscreen, Biosource, Belgium) concentrations were assessed by enzyme-linked immunoabsorbent sandwich assay (ELISA) according to manufacturers' instructions.

\section{Results}

\section{$N K$ cells play a role in the therapeutic} efficacy of intratumorally injected DC producing IL-12 Intratumoral injections of bone marrow-derived DC (BMDC) adenovirally engineered to produce IL-12 eradicate a proportion (50-80\%) of experimental colon carcinomas, including those derived from subcutaneous injection of CT26 and MC38 tumor cell lines. For those experiments immature DC were employed. The use of immature DC was preferred since artificially released BMDC inside malignant tissue had to uptake and efficiently process tumor antigens and subsequently migrate to lymph nodes. Antitumor effects required the expression of syngenic MHC molecules on $\mathrm{DC}$ and the activity of $\mathrm{CD}^{+} \mathrm{T}$ cells [5]. By contrast, depletion of $\mathrm{CD}^{+}{ }^{+} \mathrm{T}$ cells did not impair the ability of the pro- cedure to induce regressions of CT26 tumors [5]. In this study (Fig. 1), we observe that depletion of AsialoGM1 ${ }^{+}$ cells resulted in a moderate reduction of tumor regressions of subcutaneous CT26-derived experimental malignancies achieved by intratumoral injection of AdCMVIL-12-engineered BMDC ( 10 out of 15 vs 7 out of 15 cases). In addition, there was a nonstatistically significant tendency to a delay (approximately 1-2 weeks) in the time elapsed from treatment on day 7 to the beginning of tumor shrinkage in those mice depleted with anti-AsialoGM1, which eventually underwent tumor eradication (Fig. 1). It was confirmed that depletion of $\mathrm{CD}^{+} \mathrm{T}$ cells with specific mAb did not modify the efficacy (Fig. 1). With these experimental results, it seemed that NK cells played only a minor role but, surprisingly, simultaneous depletion of AsialoGM1 ${ }^{+}$and $\mathrm{CD}^{+}$ lymphocytes almost completely eliminated the antitumor activity induced by a single intratumoral injection on day 7 of tumor growth with $5 \times 10^{5}$ BMDC transfected with AdCMVIL-12 (Fig. 1).

Besides, mAb blocking IFN- $\gamma$ function completely eliminated the therapeutic effect if given to 6 mice treated intra-

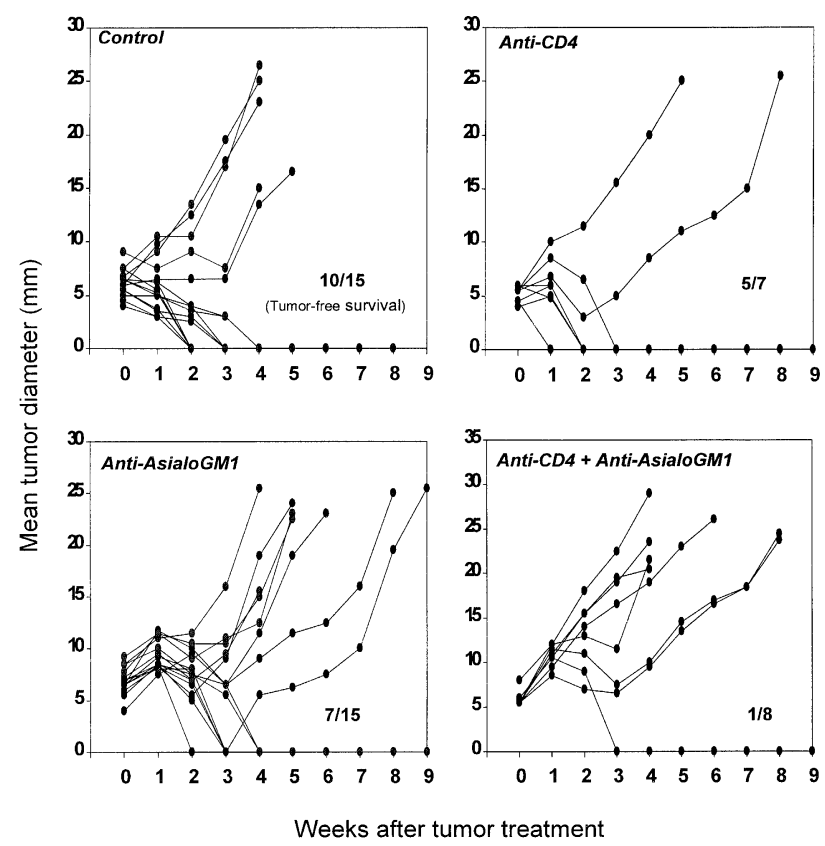

Figure 1. Combined depletion of $\mathrm{CD} 4^{+}$and AsialoGM1 ${ }^{+}$lymphocytes abrogates antitumor effects elicited by intratumoral injection of DC transfected with AdCMVmIL-12. Growth of CT26-derived experimental tumors in $\mathrm{BALB} / \mathrm{c}$ mice that were injected intraperitoneally with the indicated depleting antibodies or control rat IgG on days 5, 6, 7, 14, and 21 after tumor inoculation. All mice were treated with a single intratumoral injection of $5 \times 10^{5}$ BMDC transfected with AdCMVmIL-12 on day 7 after tumor cell challenge. Control and double-depleted groups were statistically different with a $p=0.027$ according to Fisher exact test. Efficacy of depletion was monitored by FACS analysis of peripheral blood mononuclear cells in identically treated mice (data not shown). Fraction of tumorfree mice at week 12 has been included in each panel. 


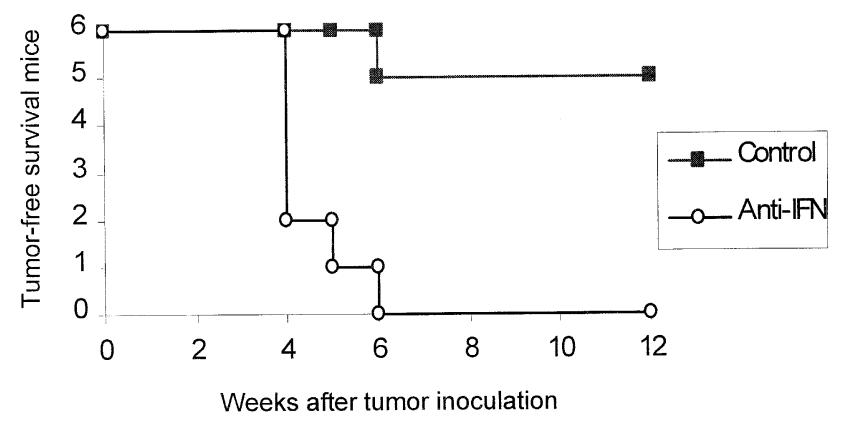

Figure 2. Inhibition of IFN- $\gamma$ function eliminates the efficacy of intratumorally injected DC secreting IL-12. Number of tumor-free surviving animals from two groups of $\mathrm{BALB} / \mathrm{c}$ mice that received $10^{5} \mathrm{DC}$ transfected with AdCMVIL-12 and injected inside CT26-derived subcutaneous tumors implanted 6 days earlier. Mice received, as indicated in the figure, on day 7 and 14 after tumor cell challenge $100 \mu \mathrm{L}$ of ascites containing anti-IFN- $\gamma$ neutralizing $\mathrm{mAb}$ or control antibody. Statistical difference according to log-rank test was $p<0.01$.

tumorally with IL-12-producing DC (Fig. 2). Those results are consistent with a major role of CTL induction in this type of immunotherapy, as well as with the prominent role of IFN- $\gamma$ as a downstream mediator of most of the effects of IL-12 [17]. We have confirmed, as previously reported by others $[6,16]$, that FITC-labeled DC injected into a subcutaneous tumor migrate to draining lymph nodes with high intensity (Fig. 3a). Moreover, some degree of migration was also found both into contralateral inguinal lymph nodes and into the spleen (M. Duarte et al., manuscript in preparation).

We have also found that intratumoral injection of IL-12-engineered DC elicits some IFN- $\gamma$ production in the draining lymph node that is partly inhibited by AsialoGM1 ${ }^{+}$depletion (Fig. 3b). This result indicates that secretion of IFN- $\gamma$ under these conditions is in part mediated by NK cells, and it is consistent with the observation of a slight upregulation of NK cytolytic activity against YAC-1 in lymph node cells that is abolished by anti-AsialoGM1 treatment (Fig. 3c). Surprisingly, the increase in NK activity was higher in splenocytes than in the cells from draining lymph nodes of the same mice (Fig. 3c). This could be related to the relative higher proportion of $\mathrm{DX} 5^{+} \mathrm{NK}$ cells in the spleen in comparison to lymph nodes as observed in control mice (not shown). It is unknown whether NK activity is elicited by those barely detectable migrating DC into the spleen or by a cytokine cascade provoked by DC producing IL-12 outside the spleen.

\section{AdCMVIL-12-engineered BMDC activate NK}

functions in vivo through a mechanism dependent on IL-12 In order to investigate whether BMDC engineered to produce IL-12 activated NK functions in vivo, experiments were carried out by direct intrasplenic injection of $2 \times 10^{6}$ BMDC in Rag1 ${ }^{-1-}$ C57BL/6 mice. These mice lack both $\mathrm{T}$ and B cells. Hence, NK cells represent the only lymphoid population remaining in their spleens.
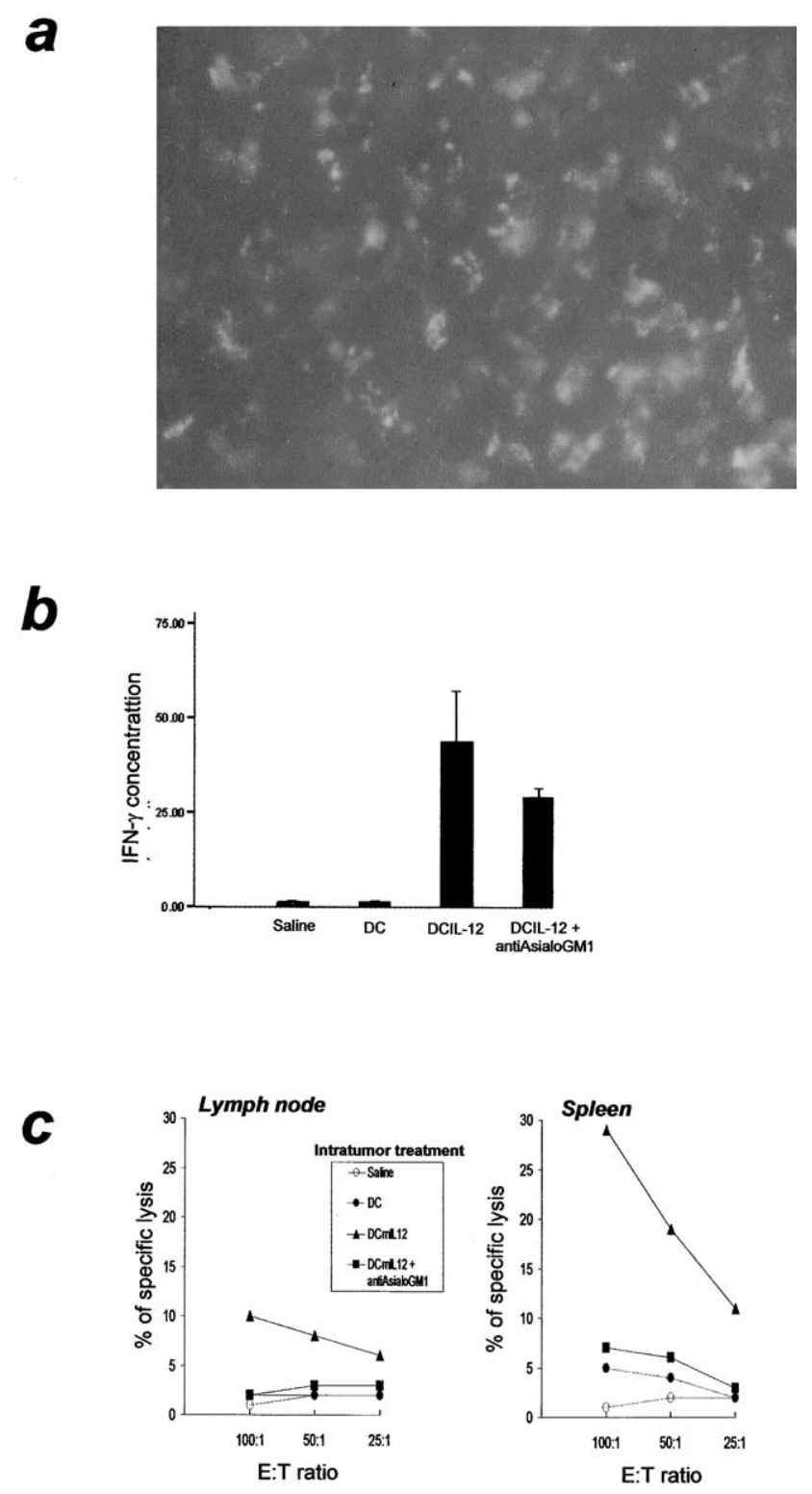

Figure 3. Intratumoral injection of IL-12-transfected DC enhances NK functions in lymphoid tissue. (a) Migration of DC assessed by a representative microscopic field $(\times 200)$ of the draining lymph node from three mice bearing subcutaneous CT26 tumors that received an intratumoral dose of $5 \times 10^{5}$ AdCMVmIL-12-transfected DC, which had been FITC-labeled prior to injection. Absence of fluorescence background in mice intratumorally injected with saline was negible. (b) IFN- $\gamma$ concentration (mean \pm SEM) assessed by ELISA in 24-hour supernatants from lymph node cell suspensions $\left(5 \times 10^{5} / \mathrm{mL}\right)$ derived from CT26 tumor-bearing BALB/c mice (three mice per group) that had been treated with intratumoral injections of HBSS, nontransduced DC, or IL-12-transduced DC with or without simultaneous depletion of AsialoGM1 ${ }^{+}$cells. For statistical analysis data from multiple experiments have been pooled and results found to be statistically different ( $p<0.01$ ) for DC vs DCIL-12 according to twotailed Student's $t$-test. (c) Cytolytic activity against NK-sensitive YAC-1 cell line detected in draining lymph nodes and in the spleen of CT26 tumor-bearing mice treated as in (b); Effector cells were harvested and pooled from three mice per group 48 hours after intratumoral treatment with DC. Using results from pooled repeated experiments, the groups with AdCMVIL-12-transfected DC were found to be statistically different ( $p<$ 0.05) using Mann-Whitney's $U$-test. 
Spleen cells from mice that had received, 24 hours earlier, a single intrasplenic injection of BMDC transfected with AdCMVIL-12, augmented their cytolytic activity against the NK-sensitive YAC-1 lymphoma and the syngenic NK-resistant MC38 colon carcinoma (Fig. 4a). By contrast, such an effect was not found when BMDC had been transfected with a control adenovirus encoding for $\beta$-galactosidase (AdCMVLacZ) (Fig. 4a). In order to find out whether the observed NK-activating properties were exclusively due to IL-12 secretion or whether other molecules expressed by DC were contributing to the effect, we performed similar experiments, injecting transfected fibroblasts instead of DC. To do so, we used equal numbers of the SV40T-transformed fibroblast cell

a

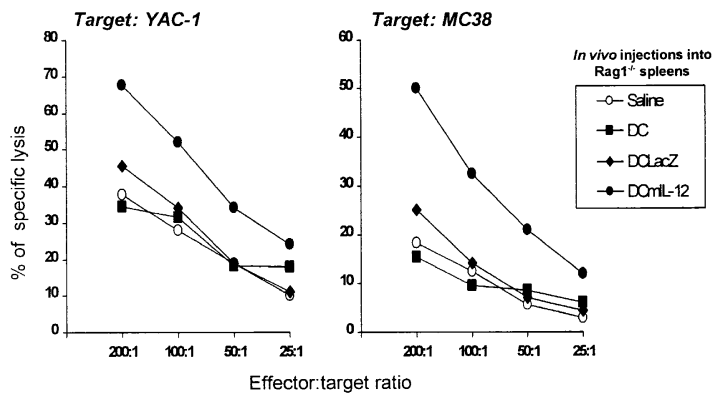

b

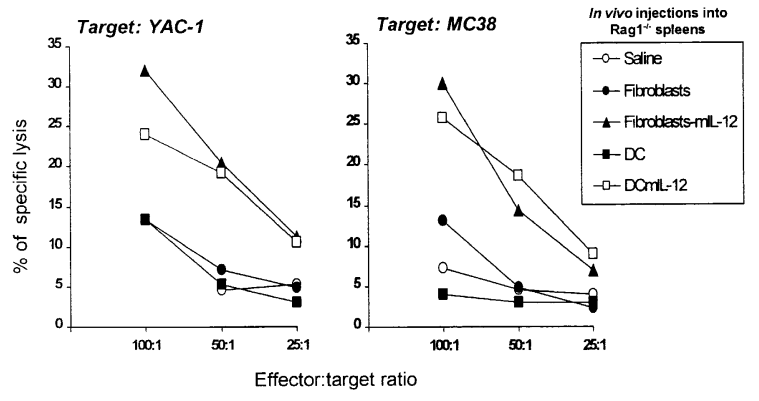

Figure 4. AdCMVmIL-12-engineered DC activate NK functions in vivo through a mechanism dependent on IL-12. (a) Rag $1^{-/-}$C57BL/6 mice (three per group) received an intrasplenic injection of AdCMVmIL-12-transfected DC, AdCMVLacZ-transfected DC, or untransfected DC $\left(2 \times 10^{6}\right.$ cells $)$. Similar mice were injected with saline buffer as a control. 24 hours later splenocytes were tested for cytolytic activity in a standard ${ }^{51} \mathrm{Cr}$-release assay against YAC-1 and MC38 cell lines at the indicated effector:target ratios. (b) $\mathrm{Rag}^{-1-}$ mice were treated with a single intrasplenic injection of either AdCMVmIL-12-transfected DC, IL-12-transfected fibroblast cell line (IZA-1), or their untransfected counterparts $\left(2 \times 10^{6}\right.$ cells in every case). A control group of similar mice received a single intrasplenic injection of saline buffer. Splenocytes were assessed 24 hours later for cytolytic activity against YAC-1 and MC38 cell lines. In vitro levels of IL-12 p70 output by transfected fibroblasts were comparable to equally transfected DC (not shown). Results are representative of three independent experiments similarly performed. Using results of the group of DC or fibroblasts transfected with IL-12, they were found to be statistically different ( $p<$ 0.05 ) than the other groups using Mann-Whitney's $U$-test. line IZA-1 [5] identically transfected with AdCMVIL-12. Our data clearly show that IL-12-transfected IZA-1 upregulated NK function to a similar extent as IL-12-BMDC did (Fig. 4b). It is noteworthy that the level of IL-12 p70 output in vitro by transfected fibroblasts was comparable to equally transfected BMDC [5]. It is also worth remembering that we have previously reported that intratumoral injection of AdCMVIL-12-transfected IZA-1 did not exert any antitumor activity [5] or induce detectable antitumor CTL.

\section{Human immature, monocyte-derived DC}

augment NK activities in vitro only if transfected with

an adenovirus encoding for human IL-12 (AdCMVhIL-12)

The results obtained by intrasplenic injections in the murine system could not be obviously confirmed as such in humans, so in vitro cocultures of DC and NK cells of human origin were performed to address this issue.

Human NK cells were obtained as described above achieving up to $95 \%$ purity encompassing $\cong 80 \% \mathrm{CD}^{-}$ $\mathrm{CD}^{+} 6^{+}$and $15 \% \mathrm{CD}^{+} \mathrm{CD}^{+} 6^{+}$lymphocytes (Fig. 5a). Human DC were derived from $\mathrm{CD}_{14}{ }^{+}$immunomagnetically selected cells by 7-day culture with GM-CSF + IL-4. Resulting cells were $\mathrm{CD} 14^{-} \mathrm{CD} 11 \mathrm{c}^{+}$as shown in Figure $5 \mathrm{~b}$.

As shown in Figure 6a, human monocyte-derived DC transfected with an adenovirus carrying both chains of human IL-12 (AdCMVhIL-12) (MOI 1000) produced important quantities of IL-12 heterodimer (p70), in contrast to untransfected DC or DC transfected with a control adenovirus.
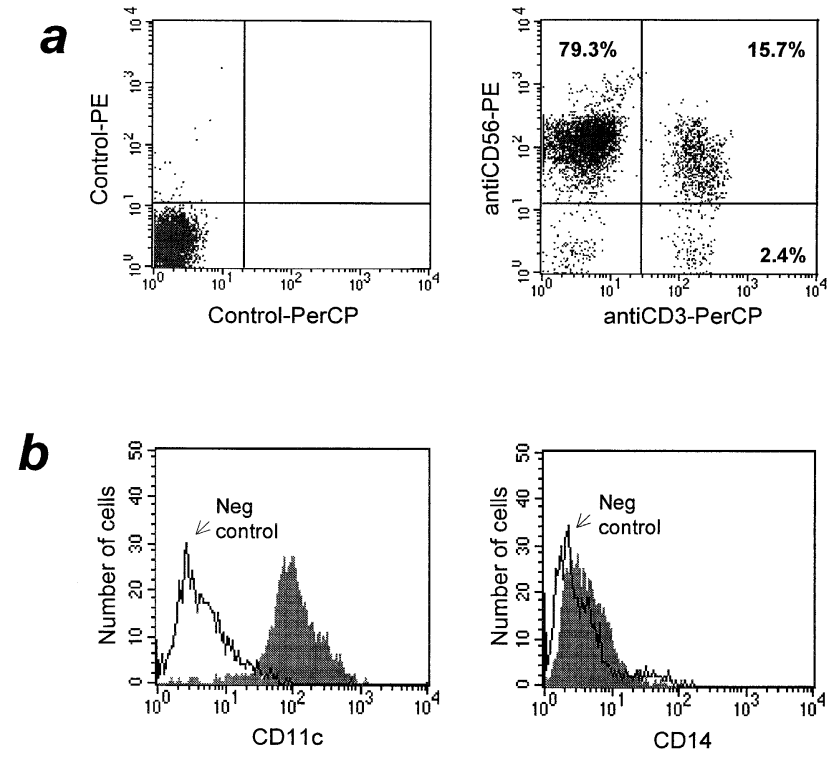

Figure 5. Enrichment of human NK cells and culture of monocyte-derived human DC. (a) $\mathrm{CD}^{+} 6^{+}$cells were purified by magnetic beads yielding $79 \%$ of $\mathrm{CD}^{-} \mathrm{CD}^{+} 6^{+}$cells as assessed by double immunostaining and flow cytometry. (b) Immunostaining and FACS analysis of $\mathrm{CD}_{14}{ }^{+}$cells purified from human PBMC by immunomagnetic beads and cultured for 7 days in the presence of GM-CSF and IL-4. 

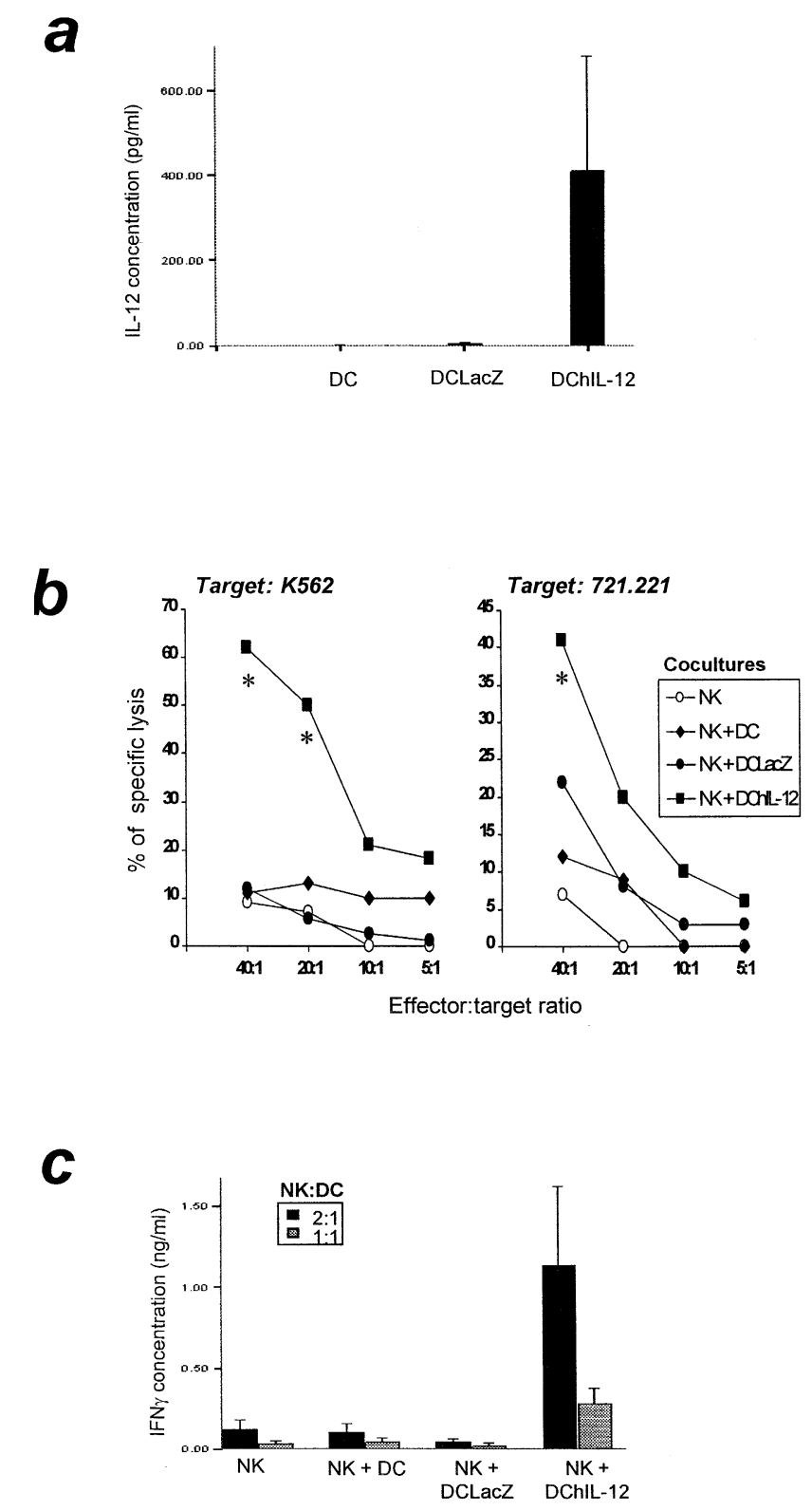

Figure 6. Human monocyte-derived DC augment NK activity in in vitro coculture only if they have been engineered to produce IL-12. (a) IL-12 p70 concentration (mean \pm SEM) assessed by ELISA at 48 hours in supernatants of human immature DC $\left(1 \times 10^{6} / \mathrm{mL}\right)$ left untransfected or transfected with LacZ or IL-12 genes. (b) Human DC, cultured for 7 days from purified $\mathrm{CD} 14^{+}$PBMC, were left untransfected or transfected either with LacZ or human IL-12 genes by recombinant adenoviruses, and subsequently cocultured with $\mathrm{CD}^{2} 6^{+}$lymphocytes. 24 hours later, cytolytic activity of cocultures against K562 and 721.221 human leukemia cell lines was comparatively assayed in standard ${ }^{51} \mathrm{Cr}$-release assays. Data are representative of five experiments similarly performed. For statistical analysis data from experiments have been pooled and results found to be statistically different $(p<0.05)$, as indicated $(*)$ for DC vs DCIL-12 according to Mann-Whitney's $U$-test. (c) IFN- $\gamma$ concentration (mean \pm SEM) quantified by ELISA in 24-hour supernatants from cocultures of DC and CD56 ${ }^{+}$ cells as described in (b) that were performed at the indicated NK:DC ratios.
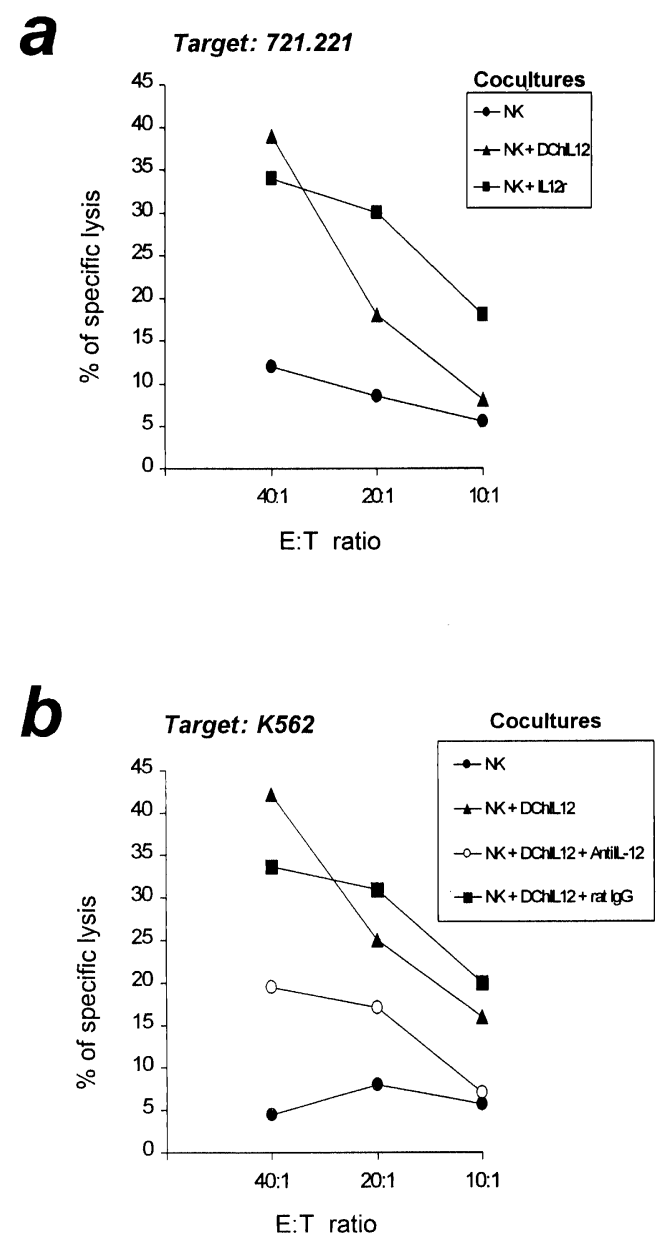

Figure 7. IL-12 as a purified protein and DC transfected with IL-12 enhance NK activity of $\mathrm{CD}^{2} 6^{+}$lymphocytes with similar intensity. (a) Interleukin-12 as a recombinant protein $(1 \mathrm{ng} / \mathrm{mL})$ was added to cultures of $\mathrm{CD}^{+} 6^{+}$cells in parallel to the coculture of IL-12-transfected DC with $\mathrm{CD} 6^{+}$lymphocytes. 24 hours later cells were tested for their cytolytic activity against the 721.221 cell line. (b) Addition of $5 \mu \mathrm{g} / \mathrm{mL}$ of anti-IL-12 p40 neutralizing antibody inhibited the increase of cytolytic activity against K562 cells of $\mathrm{CD}^{2} 6^{+}$lymphocytes that had been cocultured with AdCMVIL-12-transfected DC. Results are the mean of triplicate assays in which SD was less than $10 \%$. Polyclonal rat IgG was used as a control $(5 \mu \mathrm{g} / \mathrm{mL})$.

At this point it is worth mentioning that we and others have previously reported moderate changes in surface molecules towards maturation in adenovirally transfected DC [41,42].

It was observed that cytotoxicity against K562 and 721.221 target cells augmented after 24-hour coculture of $\mathrm{CD}^{+} 6^{+}$lymphocytes and DC (NK:DC $=4: 1,2: 1$, and 1:1). Such an effect was not sustained if DC had not been transfected with AdCMVhIL-12 and only marginally if transfected with a control adenovirus (AdCMVLacZ) (Fig. 6b).

In addition, IFN- $\gamma$ secretion was observed only when DC had been transfected with AdCMVhIL-12 but not with a control adenovirus (Fig. 6c).

These results lead to the conclusion that IL-12 is the main NK-stimulating factor in this setting. This is further 
supported by experiments with human recombinant IL-12 added to the cultures of $\mathrm{CD} 56^{+}$lymphocytes as a purified protein $(1 \mathrm{ng} / \mathrm{mL})$ that augmented NK activity in a comparable fashion to coculture with AdCMVhIL-12-transfected human DC (Fig. 7a). In addition, the induction of cytolytic activity by DC-producing IL-12 in coculture with CD56 ${ }^{+}$ cells was markedly, albeit not completely, inhibited by neutralizing anti-IL-12 mAb (Fig. 7b).

\section{Human immature DC augment}

in vivo mouse NK activity when transfected

with murine IL-12 but not with human IL-12

In an experimental setting of intrasplenic injections in $\operatorname{Rag} 1^{-1-}$ mice, as the one shown in Figure 3, human DC were also tested to see whether they upregulated NK functions. To address this issue, monocyte-derived DC were transfected either with AdCMVmIL-12 or with AdCMVhIL-12. It should be noted that human IL-12 fails to display activity on murine cells [17] and this fact let us assess whether IL-12 secretion was the unique requirement in the system. It was found that cytolytic activity in injected spleens against YAC-1 and MC38 was only significantly increased by DC-producing murine but not human IL-12 (Fig. 8). These results strongly suggest that IL-12 operates by directly increasing NK functions rather than through autocrine loops involving DC themselves. Such a possibility is ruled out by the fact that IL-12R on human DC is not sensitive to mouse IL-12.

\section{Discussion}

Interleukin-12 was originally identified as a cytokine upregulating NK functions [17]. In fact, T and NK lymphocytes

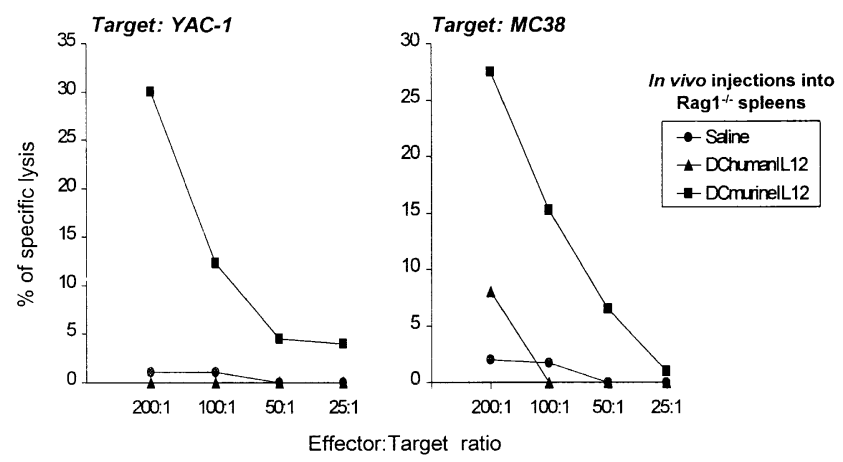

Figure 8. Human DC activate murine splenic NK cells only if transfected to produce murine but not human IL-12. Rag1 ${ }^{-l-}$ C57BL/6 mice (three per group) received intrasplenic injections of $2 \times 10^{6} \mathrm{BMDC}$ transfected with either human or murine IL-12 genes by recombinant adenoviruses (AdCMVhIL-12 or AdCMVmIL-12 as indicated). 24 hours later splenocytes were tested for cytolytic activity in a standard ${ }^{51} \mathrm{Cr}$-release assay against YAC-1 and MC38 cell lines. Results are representative of three independent experiments similarly performed. For statistical analysis data from experiments have been pooled and results found to be statistically different $(p<0.01)$ for DChumanIL-12 vs DCmurineIL-12 according to two-tailed Student's $t$-test. express the $\beta_{1} \beta_{2}$-heterodimer receptor for this cytokine, and importantly, IL-12 elicits strong antigen-independent secretion of IFN- $\gamma$ from such cells [22]. DC also express a functional alternative form of the IL-12 receptor that signals in a different way [43].

To dissect out the cellular and molecular mechanisms of the impressive antitumor efficacy shown by intratumoral injection of IL-12-transfected DC [5,6], classical antibody depletions were performed. Treatment with antiAsialoGM1, a polyclonal antiserum selective for NK and NK-T cells, slightly decreased the efficacy of intratumoral injection of IL-12-secreting BMDC. It had been previously reported that in that precise model, $\mathrm{CD}^{+} \mathrm{T}$ cells played an absolutely necessary role whereas depletion of $\mathrm{CD}^{+}$cells did not impair tumor regressions [5]. In this study we report that simultaneous depletion of $\mathrm{CD}^{+}$and AsialoGM1 ${ }^{+}$lymphocytes abrogates the tumor-eradicating immune response. Under these conditions, T-helper, NK, and NK-T cells are eliminated. It should be noted that intratumorally injected DC express neither CD4 nor AsialoGM1 and hence cannot be depleted by those antibodies.

These results indicate that a certain activity that might be performed either by NK or by T-helper cells is required for the antitumor effects. What could it be? Our results, clearly showing that blocking in vivo IFN- $\gamma$ activity by neutralizing $\mathrm{mAb}$ eliminates antitumor efficacy, together with the observations by Nishioka et al. [6] on the production of IFN- $\gamma$ in the lymphoid organs after intratumoral injection of IL-12-secreting BMDC, indicate that early antigen-independent IFN- $\gamma$ production by at least one of those cellular sources $\left(\mathrm{CD} 4^{+}\right.$or $\mathrm{NK}$ cells) is indispensable to achieve tumor regression. Our data upon depletion of AsialoGM1 ${ }^{+}$ cells support a role for NK cells in that early IFN- $\gamma$ secretion that is shown to correlate with a slight increase in their lytic activity.

It has been recently reported that NK-T cells could be key players in this system as they have been involved in IL12-mediated therapy of liver metastasis [44]. We have not explored that possibility, but experiments are to be conducted using NK-T-deficient mice [44], and it remains formally possible that our observations upon depletion with anti-AsialoGM1 are dependent on this NK-T subpopulation. However NK-T cells are quite selectively located in the liver and their involvement in immunotherapy has only been demonstrated for experimental hepatic metastasis [44].

NK involvement in antitumor immunity has been proved for acute exposure to artificially transferred malignant cells [45], but not so clearly in other settings. In immunotherapy, endogenous NK cells have been found necessary when using IL-12 as a protein [32] or as transfected gene, but it should be noted that their involvement is found in some models but not in others [17,21,46,47]. A role for those lymphoid NK subpopulations has also been found in other immunotherapeutic approaches such as in the transfection of CD80 into melanoma cells [30] or in the treatment with 
immunostimulating anti-4-1BB $\mathrm{mAb}$ [31]. Although the molecular sequence of events in which NK cells are involved is not well understood, it is doubtful that the main function carried out by NK cells could be mere tumor destruction by direct cytotoxicity, and it has been postulated that what NK cells really do is an early secretion of proinflammatory mediators required for a subsequent CTL response.

Direct cross-talk of DC and NK cells has been proposed. Initial observations came from the fact that NK cells are involved in the antitumor effects elicited by treatment with Flt3L [33]. This molecule induces not only the accumulation of dendritic cells infiltrating tumors and the induction of CTL [48], but also a marked augment on NK activity $[33,49]$. Using a mature human DC line, it was shown that those human DC could activate mouse NK cells to acquire cytolytic activity and IFN- $\gamma$ secretion capacity [33]. The molecular mechanism, according to this previous report, involves close membrane-to-membrane contact [33]. It is worth mentioning in this regard that functionally inducible membrane-associated IL-12 on DC has been reported [50]. DC-NK cross-talk through cytokines has been recently confirmed with human DC and NK cells derived in vitro from cord blood CD34 ${ }^{+}$cells [34]. In this case an involvement of IL-12, IL-18, and possibly IFN- $\gamma$ produced by DC was unraveled, showing also a possible role of DC-expressed CD40 acting through an unknown counterreceptor on NK cells distinct from CD40L [34]. In both pieces of work, DC were matured with TNF- $\alpha$ prior to exposure to NK cells. In our system, we also found moderate upregulation of NK functions by DC if they had been matured with TNF- $\alpha$ (not shown) but not if left immature. In the present study we have used a novel system to analyze the effects of DC in vivo by direct injections into the spleen of B- and T-cell-deficient mice that has been very informative on these issues. With these experiments, it was found that when immature DC are used by intrasplenic injection or in in vitro coculture, they do not activate NK cells. Under our conditions, NK activation was induced strictly and directly by IL- 12 .

The most relevant findings of this study are that 1) AsialoGM1 ${ }^{+}$cells play an important role in tumor rejections elicited by immature IL-12-producing DC injected inside malignant tissue, and that 2) immature DC can activate NK functions both in vivo and in vitro only if they have been previously gene-modified to produce IL-12. Interestingly, as mentioned before, IL-12-producing fibroblasts injected in the spleen of Rag $1^{-1-}$ mice also upregulated NK functions but failed to induce tumor regressions if injected intratumorally in the same range of doses [5]. The main difference between those cell types in this regard is that $\mathrm{BMDC}$, in contrast to fibroblasts, intensely migrate from tumor to lymphoid tissue [16]. It can be inferred from our results that we have experimentally bypassed the need for migration by direct intrasplenic injections. In addition, fibroblasts perform poorly as antigen-presenting cells [51], although this point has been the matter of some debate in the past [52] and some efficacy has been found by peritumoral injection of IL-12-secreting fibroblasts [53] and confirmed in a recent clinical trial.

Dissecting out the intimate mechanisms involved in intratumoral gene therapy with DC engineered to produce IL-12 might be difficult. NK activation joins now the alreadyknown requirements of tumor antigen uptake, DC migration to lymphoid organs, and the orchestration of a potent CTLmediated immune response as recently suggested $[5,6,11,54]$. The role of NK cells could be double either through early cytotoxicity causing release of tumor antigens, thus making them available for DC-mediated presentation, or through proinflammatory cytokine/chemokine secretion, as found in this study, by the AsialoGM1-sensitive production of IFN- $\gamma$ detected in lymphocytes from explanted draining lymph nodes. If this is the case, artificial potentation of both NK activities could enhance the efficacy achieved by this promising DC-based immunotherapeutic approach, which is entering clinical trials at our institution and elsewhere.

\section{Acknowledgments}

Financial support was from CICYT (SAF99-0039) and from Gobierno de Navarra (Beca Ortiz de Landázuri). MR-C is the recipient of a fellowship from Fundación Española de Hematología y Hemoterapia.

We are also grateful to Miguel López-Botet for the critical reading of the manuscript, for the generous gift of reagents and cell lines, and for helpful suggestions. Stimulating scientific discussions and long-term collaborations with Maurizio Bendandi, Felipe Prósper, José Rifón, Juan Ruiz, Juanjo Lasarte, Pablo Sarobe, Francisco Borrás, and Eduardo Rocha are also acknowledged, as well as excellent technical support by Izaskun Gabari, Helena Villanueva, and Juan Percaz.

\section{References}

1. Banchereau J, Briere F, Caux C, et al. (2000) Immunobiology of dendritic cells. Annu Rev Immunol 18:767

2. Fong L, Engleman EG (2000) Dendritic cells in cancer immunotherapy. Annu Rev Immunol 18:245

3. Timmerman JM, Levy R (1999) Dendritic cell vaccines for cancer immunotherapy. Annu Rev Med 50:507

4. Steinman RM, Pack M, Inaba K (1997) Dendritic cell development and maturation. Adv Exp Med Biol 417:1

5. Melero I, Duarte M, Ruiz J, et al. (1999) Intratumoral injection of bone marrow-derived dendritic cells engineered to produce interleukin-12 induces complete regression of established murine transplantable colon adenocarcinomas. Gene Ther 6:1779

6. Nishioka Y, Hirao M, Robbins PD, Lotze MT, Tahara H (1999) Induction of systemic and therapeutic antitumor immunity using intratumoral injection of dendritic cells genetically modified to express interleukin 12. Cancer Res 59:4035

7. Miller PW, Sharma S, Stolina M, et al. (2000) Intratumoral administration of adenoviral interleukin 7 gene-modified dendritic cells augments specific antitumor immunity and achieves tumor eradication. Hum Gene Ther 11:53 
8. Furumoto K, Arii S, Yamasaki S, et al. (2000) Spleen-derived dendritic cells engineered to enhance interleukin-12 production elicit therapeutic antitumor immune responses. Int J Cancer 87:665

9. Kikuchi T, Moore MA, Crystal RG (2000) Dendritic cells modified to express CD40 ligand elicit therapeutic immunity against preexisting murine tumors. Blood 96:91

10. Larsson M, Fonteneau JF, Bhardwaj N (2001) Dendritic cells resurrect antigens from dead cells. Trends in Immunol 22:141

11. Melero I, Vile RG, Colombo MP (2000) Feeding dendritic cells with tumor antigens: self-service buffet or a la carte? Gene Ther 7:1167

12. Russo V, Zhou D, Sartirana C, et al. (2000) Acquisition of intact allogeneic human leukocyte antigen molecules by human dendritic cells. Blood 95:3473

13. Suto R, Srivastava PK (1995) A mechanism for the specific immunogenicity of heat shock protein-chaperoned peptides. Science 269:1585

14. Castellino F, Boucher PE, Eichelberg K, et al. (2000) Receptor-mediated uptake of antigen/heat shock protein complexes results in major histocompatibility complex class I antigen presentation via two distinct processing pathways. J Exp Med 191:1957

15. Binder RJ, Anderson KM, Basu S, Srivastava PK (2000) Cutting edge: heat shock protein gp96 induces maturation and migration of $\mathrm{CD} 11 \mathrm{c}^{+}$ cells in vivo. J Immunol 165:6029

16. Hirao M, Onai N, Hiroishi K, et al. (2000) CC chemokine receptor-7 on dendritic cells is induced after interaction with apoptotic tumor cells: critical role in migration from the tumor site to draining lymph nodes. Cancer Res 60:2209

17. Trinchieri G (1998) Interleukin-12: a cytokine at the interface of inflammation and immunity. Adv Immunol 70:83

18. Yokoyama W (1999) Natural killer cells. In: WE Paul (ed), Fundamental Immunology. Philadelphia, PA: Lippincott-Raven, p. 575

19. Perussia B, Chan SH, D'Andrea A, et al. (1992) Natural killer (NK) cell stimulatory factor or IL-12 has differential effects on the proliferation of TCR- $\alpha \beta^{+}$, TCR $-\gamma \delta^{+}$T lymphocytes, and NK cells. J Immunol 149:3495

20. Soiffer RJ, Robertson MJ, Murray C, Cochran K, Ritz J (1993) Interleukin-12 augments cytolytic activity of peripheral blood lymphocytes from patients with hematologic and solid malignancies. Blood 82:2790

21. Soloski MJ (2001) Recognition of tumor cells by the innate immune system. Curr Opin Immunol 13:154

22. Azzoni L, Kanakaraj P, Zatsepina O, Perussia B (1996) IL-12-induced activation of $\mathrm{NK}$ and $\mathrm{T}$ cells occurs in the absence of immediate-early activation gene expression. J Immunol 157:3235

23. Moretta A, Biassoni R, Bottino C, Mingari MC, Moretta L (2000) Natural cytotoxicity receptors that trigger human NK cell-mediated cytolysis. Immunol Today 21:228

24. Ravetch JV, Lanier LL (2000) Immune inhibitory receptors. Science 290:84

25. Lopez-Botet M, Perez-Villar JJ, Carretero M, Rodriguez A, Melero I (1996) Functional resemblance between the Ig-related NK cell receptors specific for HLA class I molecules and the CD94 C-type lectin. Chem Immunol 64:116

26. Leibson PJ (1997) Signal transduction during natural killer cell activation: inside the mind of a killer. Immunity 6:655

27. Kennedy MK, Glaccum M, Brown SN, et al. (2000) Reversible defects in natural killer and memory CD8 T-cell lineages in interleukin 15deficient mice. J Exp Med 191:771

28. Biron CA, Byron KS, Sullivan JL (1989) Severe herpesvirus infections in an adolescent without natural killer cells. N Engl J Med 320:1731

29. Gilmour KC, Fujii H, Cranston T, Davies EG, Kinnon C, Gaspar HB (2001) Defective expression of the interleukin-2/interleukin-15 receptor subunit leads to a natural killer cell-deficient form of severe combined immunodeficiency. Blood 98:877

30. Wu TC, Huang AY, Jaffee EM, Levitsky HI, Pardoll DM (1995) A reassessment of the role of B7-1 expression in tumor rejection. J Exp Med 182:1415

31. Melero I, Johnston JV, Shufford WW, Mittler RS, Chen L (1998)
NK1.1 cells express 4-1BB (CDw137) costimulatory molecule and are required for tumor immunity elicited by anti-4-1BB monoclonal antibodies. Cell Immunol 190:167

32. Kodama T, Takeda K, Shimozato O, et al. (1999) Perforin-dependent NK cell cytotoxicity is sufficient for anti-metastatic effect of IL-12. Eur J Immunol 29:1390

33. Fernandez NC, Lozier A, Flament C, et al. (1999) Dendritic cells directly trigger $\mathrm{NK}$ cell functions: cross-talk relevant in innate anti-tumor immune responses in vivo. Nat Med 5:405

34. Yu Y, Hagihara M, Ando K, et al. (2001) Enhancement of human cord blood CD $34^{+}$cell-derived NK cell cytotoxicity by dendritic cells. J Immunol 166:1590

35. Mazzolini G, Narvaiza I, Perez-Diez A, et al. (2001) Genetic heterogeneity in the toxicity to systemic adenoviral gene transfer of interleukin12. Gene Ther 8:259

36. Inaba K, Inaba M, Romani N, et al. (1992) Generation of large numbers of dendritic cells from mouse bone marrow cultures supplemented with granulocyte/macrophage colony-stimulating factor. J Exp Med 176:1693

37. Sallusto F, Lanzavecchia A (1994) Efficient presentation of soluble antigen by cultured human dendritic cells is maintained by granulocyte/macrophage colony-stimulating factor plus interleukin 4 and downregulated by tumor necrosis factor- $\alpha$. J Exp Med 179:1109

38. Mazzolini G, Qian C, Xie X, et al. (1999) Regression of colon cancer and induction of antitumor immunity by intratumoral injection of adenovirus expressing interleukin-12. Cancer Gene Ther 6:514

39. Qian C, Bilbao R, Bruna O, Prieto J (1995) Induction of sensitivity to ganciclovir in human hepatocellular carcinoma cells by adenovirusmediated gene transfer of herpes simplex virus thymidine kinase. Hepatology 22:118

40. Melero I, Salmeron A, Balboa MA, Aramburu J, Lopez-Botet M (1994) Tyrosine kinase-dependent activation of human NK cell functions upon stimulation through a $58-\mathrm{kDa}$ surface antigen selectively expressed on discrete subsets of NK cells and T lymphocytes. J Immunol 152:1662

41. Duarte M, Boya P, Rodríguez-Calvillo M, et al. (2001) Dendritic cells infected with recombinant defective adenoviruses undergo NF- $\mathrm{KB}$ activation and partial maturation. Inmunología 20:130

42. Morelli AE, Larregina AT, Ganster RW, et al. (2000) Recombinant adenovirus induces maturation of dendritic cells via an NF-кB-dependent pathway. J Virol 74:9617

43. Grohmann U, Belladonna ML, Bianchi R, et al. (1998) IL-12 acts directly on DC to promote nuclear localization of NF- $\mathrm{KB}$ and primes DC for IL-12 production. Immunity 9:315

44. Cui J, Shin T, Kawano T, et al. (1997) Requirement for V $\alpha 14$ NKT cells in IL-12-mediated rejection of tumors. Science 278:1623

45. Kim S, Iizuka K, Aguila HL, Weissman IL, Yokoyama WM (2000) In vivo natural killer cell activities revealed by natural killer cell-deficient mice. Proc Natl Acad Sci U S A 97:2731

46. Shurin MR, Esche C, Peron JM, Lotze MT (1997) Antitumor activities of IL-12 and mechanisms of action. Chem Immunol 68:153

47. Barajas M, Mazzolini G, Genove G, et al. (2001) Gene therapy of orthotopic hepatocellular carcinoma in rats using adenovirus coding for interleukin 12. Hepatology 33:52

48. Lynch DH, Andreasen A, Maraskovsky E, Whitmore J, Miller RE, Schuh JC (1997) Flt3 ligand induces tumor regression and antitumor immune responses in vivo. Nat Med 3:625

49. McKenna HJ, Stocking KL, Miller RE, et al. (2000) Mice lacking flt3 ligand have deficient hematopoiesis affecting hematopoietic progenitor cells, dendritic cells, and natural killer cells. Blood 95:3489

50. Quinones M, Ahuja SK, Melby PC, Pate L, Reddick RL, Ahuja SS (2000) Preformed membrane-associated stores of interleukin (IL)-12 are a previously unrecognized source of bioactive IL-12 that is mobilized within minutes of contact with an intracellular parasite. J Exp Med 192:507

51. Boussiotis VA, Barber DL, Lee BJ, Gribben JG, Freeman GJ, Nadler 
LM (1996) Differential association of protein tyrosine kinases with the T-cell receptor is linked to the induction of anergy and its prevention by B7 family-mediated costimulation. J Exp Med 184:365

52. Kundig TM, Bachmann MF, DiPaolo C, et al. (1995) Fibroblasts as efficient antigen-presenting cells in lymphoid organs. Science 268: 1343 53. Zitvogel L, Tahara H, Robbins PD, et al. (1995) Cancer immunother- apy of established tumors with IL-12. Effective delivery by genetically engineered fibroblasts. J Immunol 155:1393

54. Tanaka F, Hashimoto W, Okamura H, Robbins PD, Lotze MT, Tahara $\mathrm{H}$ (2000) Rapid generation of a potent and tumor-specific cytotoxic T lymphocyte by interleukin 18 using dendritic cells and natural killer cells. Cancer Res 60:4838 